\title{
Mycosis fungoides - a case report
}

\author{
Łukasz Michalecki, Grzegorz Głowacki, Hubert Urbańczyk, Dorota Gabryś
}

Department of Radiotherapy, Maria Sklodowska-Curie Memorial Cancer Center and Institute of Oncology, Gliwice Branch, Poland

\begin{abstract}
Mycosis fungoides (MF) is a form of T-cell lymphoma that initially manifests itself in the skin and may mimic other skin conditions related to skin aging in postmenopausal women. In some patients, extra-cutaneous dissemination may occur, usually to the lymph nodes, spleen, bone marrow, and other organs. It develops gradually from patches to more infiltrated plaques and eventually to tumors.

Although our understanding of the etiopathogenesis of this disease is still incomplete, due to the recent introduction of some sophisticated therapeutic methods, we are able to achieve remission of variable duration with minimization or even elimination of symptoms.

This report documents a case of an 82-year-old female patient, with histologically proven mycosis fungoides, who was treated at the Department of Radiation Oncology, Cancer Centre in Gliwice, Poland, with two courses of total skin electron therapy. The report presents some difficulties with early diagnosis and treatment, as well as the effectiveness of the clinicians' perseverance in the management of such an unpredictable disease.

Key words: mycosis fungoides, T-cell lymphoma, radiotherapy, total skin irradiation, primary cutaneous lymphoma.
\end{abstract}

\section{Introduction}

Women in the perimenopausal period may complain of a rash and dry, scaly or itchy skin, but we cannot ignore any symptoms and should take into account different variants of etiology. Estrogen, in particular its low concentration, together with the decreasing number of estrogen receptors in the skin, is believed to be responsible for the skin changes in the postmenopausal period. Systemic estrogens have positive effects on hormonal aging, increasing skin collagen content, thickness, elasticity and hydration. However, there are also non-hormonal causes of skin disorders such as diabetes mellitus, skin neoplasms, medicines' side effects, allergies and other uncharacteristic skin conditions, diagnosis of which may create some problems [1]. The incidence of skin lymphomas increases with age (median age at diagnosis is 50) with a fourfold increase in incidence in patients over 70 , while it is rarely seen in children and young adults [2,3]. Therefore it is important to take into account different variants and subtypes of skin lymphoma in differential investigation in perimenopausal women with nonspecific skin changes.

Mycosis fungoides (MF) is the most common primary cutaneous lymphoma, and represents nearly $50 \%$ of all primary cutaneous lymphomas, with a male-tofemale incidence ratio of $1.66[4,5]$. Mycosis fungoides is a primary form of skin lymphoma, characterized by malignant T cell spread of the lymphatic system. The disease is insidious in onset. Mycosis fungoides has an indolent clinical course with slow progression over years or sometimes decades. It develops gradually from patches to more infiltrated plaques and eventually to tumors. In some patients, lymph nodes and visceral organs may become involved by $T$ lymphocytes in the later stages of the disease. Despite its indolent course, even at low stages of disease, patients suffer from pruritus and desquamation. Patients with tumor-stage MF characteristically show a combination of patches, plaques, erythrodermia and tumors, which often show ulceration $[4,6,7]$. It was shown that advanced skin $(T)$ stage, peripheral blood involvement, increased lactate dehydrogenase (LDH), and folliculotropic MF were independent factors predictive for higher risk of disease progression and shorter overall survival [8].

Mycosis fungoides needs to be differentiated from erythematous lesions, psoriasis, or Hodgkin's disease. Morphologically, the neoplastic lymphoid infiltrate is epidermotropic and composed predominantly of atypical lymphocytes with enlarged hyperchromatic, cerebriform nuclei and clear cytoplasm [3]. It is a clonal disorder of memory T-helper cells, in which progressive immunodeficiency occurs, and consequently, patients may develop severe infections in the advanced stages [3]. The diagnosis is made on the integration of clinical, histopathologic, immunopathologic and molecular bio- 
logical characteristics. However, in many cases, there are problems finding a definitive diagnosis.

The disease is not easy to treat, but it is possible to achieve remission of different duration as well as to minimize or even eliminate the symptoms, allowing patients to live for many years [7]. Treatment of the early stages includes phototherapy with UVA, PUVA, topical corticosteroids, topical chemotherapy (nitrogen mustard, carmustine), topical retinoids (bexarotene, tazarotene), topical imiquimod and hexaroten, and total skin irradiation (TSI). In an advanced clinical stage, systemic treatment with extracorporeal photopheresis (ECP), interferons, systemic retinoids, or histone deacetylase (HDAC) inhibitors are preferred to traditional chemotherapy for patients who are not responding to initial skin-directed therapies. Multiagent chemotherapy is applied only to patients who do not respond to multiple prior therapies $[6,9]$.

\section{Case report}

This article presents a clinical case of an 82-year-old female patient, treated for mycosis fungoides for many years. This case report illustrates difficulties in the early diagnostic work-up and treatment process, and also, it highlights the effectiveness of the clinicians' perseverance in the management of such a highly unpredictable disease.

The first signs of skin rash, located on one hand, were noticed by the patient in 1989. Subsequently, she was referred by her primary care physician to the Dermatology Department, where she was treated with Grenz ray therapy (Bucky's method), antibiotics, and anti-histamine medications. The patient's skin rash resolved, and her remission lasted until 1996, when a systemic skin rash, in the form of discolored and nodular lesions, associated with intensive pruritus, occurred. A histopathological examination of the skin biopsy confirmed the premycotic stage of mycosis fungoides. Intensive treatment with steroids (Encorton $30 \mathrm{mg} / \mathrm{d}$, Polcortolon $90 \mathrm{mg} 2 \times / \mathrm{d}$ ), and PUVA therapy was started, resulting in a mild to moderate regression of the skin rash. During subsequent hospitalizations, immunosuppressive medications (Imuran 100 mg/d and Endoxan $100 \mathrm{mg} / \mathrm{d}$ ) were also added to the treatment regimen.

At the beginning of 2004 the immunohistochemical examination of the skin biopsy specimen revealed lymphoid infiltrates of mixed character, one third CD20(+) and the remainder $\mathrm{CD} 3(+)$, with predominance of CD4 over CD8, and lack of plasmatic granulocytes. In this way, the entire picture was rather supportive of a reactive lymphatic infiltrate - pseudolymphoma. Due to that finding, the patient was qualified for systemic immunosuppressive treatment which was continued with breaks until 2007.
In April 2007, due to the progression of symptoms in the form of redness and itching of the affected skin, another skin biopsy specimen was taken for pathological examination. Microscopic verification of skin lesions, from the abdominal region, allowed us to diagnose MF unequivocally. The patient was qualified for chemotherapy with chlorambucil. However, despite receiving intravenous chemotherapy cycles, her symptoms worsened (pruritus, and progressive, malignant disease spread within the skin of the trunk and lower extremities). The patient was referred to the Radiotherapy Department for sequential electron beam radiotherapy TSI. The total planned dose was $32 \mathrm{~Gy}$, administered in two 8-day series, with a 14-day break between, with 2 Gy fraction dose.

As a result of this treatment, complete regression of the skin lesions and resolution of previously intractable pruritus were achieved.

The remission in the form of small discolored skin lesions (the only abnormality) lasted for over 12 months. In August 2008, another recurrence of the neoplastic process and worsening of the disease symptoms occurred. The systemic treatment (chlorambucil) used at that time again did not induce any regression. On the contrary, there was further progress of the disease, and deterioration of the patient's general condition.

At the beginning of 2009 the patient was referred for another cycle of electron beam radiotherapy (6 MeV), based on the following modified scheme: $12 \mathrm{~Gy}$, 2-week break and $12 \mathrm{~Gy}$. The total combined dose, deposited within the entire body skin, was 24 Gy. At the end of treatment the patient experienced a mild post-radiation reaction presenting as edema, redness and pain, especially in the hands and feet. The symptoms were managed pharmacologically.

During a follow-up visit, remission of the skin lesions was found. There were telangiectasia and mild fibrosis of the skin, and she suffered from pruritus. In June 2013 she was operated on due to squamous cell carcinoma G2 of the skin. The tumor was located in the abdominal part. Her last visit to our center was in October 2013.

\section{Discussion}

Accurate diagnosis of MF is essential for staging, prognostic stratification, and determining therapeutic options. It is not uncommon for the diagnosis of MF to remain elusive for a long time, requiring observation and repeated biopsies with clinicopathological correlation. We could suspect this disease when the patient presents a long (years) history of unmanageable, recurrent, pruritic skin eruption with poikilodermatous or polymorphic skin involvement [10]. On the other hand, it could overlap clinicopathologic findings with various reactive dermatoses as well as conflicting clinical pres- 
entations and pathologic features [3]. Also in our case, we encountered problems with the proper diagnosis, and the final diagnosis was made years after the first changes of the skin appeared.

Many patients are treated with multiple therapies in their lifetime due to the chronicity of the disease. These therapies include: skin-directed therapies, such as ultraviolet light, topicals, and radiation; a large group of systemic agents ranging from retinoids through other biologics to chemotherapy; and an increasing role for allogeneic stem cell transplantation [10]. However, the treatment individualization, based on appropriate matching of the therapeutic strategies with clinical stage, and with the patient's complaints, plays the most important role in a successful outcome.

In the current case the patient was treated with systemic therapy, Grenz ray therapy and radiotherapy. Through the use of systemic therapy we could control the disease for many years. At a later stage of the disease, radiotherapy was able to provide a 12-month complete remission and re-irradiation led to another remission. In fact, TSI represents a necessary tool in the armamentarium of the oncologist managing the care of patients diagnosed with MF. Response rates after radiotherapy vary up to $97 \%$ depending on the stage of the disease [11-13].

Irradiating the total area of skin even with electrons in the majority of cases causes no severe side effects. They commonly include soreness, erythema, desquamation, cutaneous ulceration, alopecia, and separation of finger-nails from their nail bed (onycholysis). Chronically, the occurrence of telangiectasia and nail atrophy is possible $[11,14]$. Moreover, a repeated course of TSI is very rarely used, but is technically feasible and tolerable. When the dose in the second course was reduced, long-term toxicities were mild in severity and generally consisted of generalized xerosis, scattered telangiectasias, pigmentation changes, and partial alopecia [15]. In our study, the patient suffered from telangiectasia and mild fibrosis of the skin.

Another distinguishing factor for this group of patients is the appearance of a second squamous skin cancer $[6,11]$. Second malignancy in MF is a recognized phenomenon and usually occurs after potentially carcinogenic therapy [16]. On the other hand, the unique feature of cutaneous $T$ cell lymphoma is that the malignant $T$ cell clone expands at the expense of normal $T$ cells, creating an immunodeficiency, so patients with cutaneous $T$ cell lymphoma have an increased risk of second malignancies such as multiple squamous cell carcinomas, and can die due to bacterial infections and septicemia or viral infections $[6,11,16]$. Our case also confirms these results, since our patient was diagnosed with squamous cell carcinoma. Taking into account the difficulties in diagnosis, variable stages and clinicopathological features of the disease, interdisciplinary management plays an important role in the proper treatment of mycosis fungoides.

\section{Disclosure}

Authors report no conflict of interest.

\section{References}

1. Archer D.F. Postmenopausal skin and estrogen. Gynecol Endocrinol 2012; 28 Suppl 2: 2-6.

2. Wilcox R. Cutaneous T-cell lymphoma: 2014 Update on diagnosis, riskstratification, and management. Am J Hematol 2014; 89: 837-51.

3. Song SX, Willemze R, Swerdlow SH, et al. Mycosis fungoides: report of the 2011 Society for Hematopathology/European Association for Haematopathology workshop. Am J Clin Pathol 2013; 139: 466-90.

4. Willemze R, Jaffe ES, Burg G, et al. WHO-EORTC classification for cutaneous lymphomas. Blood 2005; 105: 3768-85.

5. Bradford PT, Devesa SS, Anderson WF, et al. Cutaneous lymphoma incidence patterns in the United States: a population-based study of 3884 cases. Blood. 2009; 113: 5064-73.

6. Mazzeo E, Rubino L, Buglione M, et al. The current management of mycosis fungoides and Sezary syndrome and the role of radiotherapy: Principles and indications. Rep Pract Oncol Radiother 2014; 19: 77-91.

7. Kazmierska J. Clinical results of the total skin electron irradiation of the mycosis fungoides in adults. Conventional fractionation and low dose schemes. Rep Pract Oncol Radiother 2013; 19: 99-103.

8. Agar NS, Wedgeworth E, Crichton S, et al. Survival outcomes and prognostic factors in mycosis fungoides/Sézary syndrome: validation of therevised International Society for Cutaneous Lymphomas/European Organisation for Research and Treatment of Cancer staging proposal. J Clin Oncol 2010; 28: 4730-9.

9. Dugas-Breit S, Schulze HJ, Hallermann C. New and established treatment options for mycosis fungoides and Sezary syndrome - an update. J Dtsch Dermatol Ges 2014; 12: 561-9.

10. Al Hothali GI. Review of the treatment of mycosis fungoides and Sezary syndrome: A stage-based approach. Int J Health Sci (Qassim) 2013; 7: 220-39.

11. Marta GN, Gouvêa CB, Ferreira SB, et al. Mycosis fungoides: case report treated with radiotherapy. An Bras Dermatol 2011; 86: 561-4.

12. Le Bourgeois JP, Haddad E, Marinello G, et al. The indications for total cutaneous electron beam radiation therapy of mycosis fungoides. Int J Radiat Oncol Biol Phys 1987; 13: 189-93.

13. Moraes FY, Carvalho Hde A, Hanna SA, et al. Literature review of clinical results of total skin electron irradiation (TSEBT) of mycosis fungoides in adults. Rep Pract Oncol Radiother 2013; 19: 92-8

14. Hoppe RT. Mycosis fungoides: radiation therapy. Dermatol Ther 2003; 16: 347-54.

15. Becker M, Hoppe RT, Knox SJ. Multiple courses of high-dose total skin electron beam therapy in the management of mycosis fungoides. Int J Radiat Oncol Biol Phys 1995; 32: 1445-9.

16. Le K, Lim A, Samaraweera U, et al. Multiple squamous cell carcinomas in a patient with mycosis fungoides. Australas J Dermatol 2005; 46: 270-3. 